\title{
Simplified stepwise management of primary spontaneous pneumothorax: a pilot study
}

\author{
C-H. Marquette, A. Marx, S. Leroy, F. Vaniet, P. Ramon, S. Caussade, N. Smaiti, \\ C. Bonfils, and the Pneumothorax Study Group
}

ABSTRACT: In primary spontaneous pneumothorax (PSP) requiring intervention, removal of air from the pleural space can be achieved by manual needle aspiration or by pleural drainage after insertion of a chest tube. This study aimed to evaluate the efficacy and safety of a serial-steps approach with a single system (small-calibre catheter/Heimlich valve) in a homogeneous population of patients with a first episode of PSP.

All patients had an $8.5 \mathrm{~F}$ distally multiperforated polyurethane pigtail catheter inserted via the anterior route, at the bedside without any guidance, using the Seldinger technique. A one-way Heimlich valve was connected to the catheter, allowing the air to flow spontaneously outwards for 24-48 $\mathrm{h}$. Thereafter, if the lung failed to re-expand wall suction was applied. Patients with an air leak persisting for $>4$ days were referred for surgery. Primary end-points were success rates at $24 \mathrm{~h}$ and 1 week, and $1 \mathrm{yr}$ recurrence-free survival.

In total, 41 consecutive patients entered the study. They were typically thin, young, smoking males. No procedure-related complications were observed. The need for pain relief medication was minimal. Within the first $24 \mathrm{~h}$, most of the patients were able to walk around and half of them were able to take a shower with their catheter in place. The 24-h and 1-week success rates were 61 and $85 \%$, respectively, and the actuarial 1 -yr recurrence rate was $24 \%$. Four patients underwent surgical pleurodesis because of a persistent air leak. The length of stay when excluding the four surgical cases was $2.3 \pm 1.3$ days.

When 24-h and 1-week success rates and recurrence at 12 months were taken as end-points, the method described here is as effective as simple manual needle aspiration or a conventional chest tube thoracotomy. These results support further studies assessing this "one system, serialsteps approach" in an outpatient management algorithm.

\section{KEYWORDS: Drainage, pleura, pneumothorax, surgery, treatment}

$\mathbf{P}$ rimary spontaneous pneumothorax (PSP), a common disease in daily pulmonary practice, has an estimated incidence of 7.4 out of 100,000 for males and 1.2 out of 100,000 for females (age-adjusted incidence) and usually occurs during the second or third decade in healthy "active" young people $[1,2]$.

Primary therapeutic goals for PSP include removal of air from the pleural space and prevention of recurrences. The recent guidelines of the British Thoracic Society (BTS) and the American College of Chest Physicians (ACCP) recommend that small PSP in patients without breathlessness should be observed for 3-6 $\mathrm{h}$ and

For editorial comments see page 448 . discharged on the provision that a repeat chest radiograph excludes progression over time; only in large or symptomatic PSP should air evacuation be proposed [3-5].

The absence of generally accepted and methodologically sound recommendations may account for the extensive variation in practice for air evacuation techniques, reported in observational studies $[4,6]$. Air evacuation may be achieved by simple manual needle aspiration (MA), by a small bore tube or a conventional chest tube, the tubes being attached to a one-way valve or to a water-seal device, with or without active suctioning. Currently chest tube thoracotomy remains by far, with some exceptions [7], the most popular technique $[6,8,9]$.

\section{AFFILIATIONS}

Clinique des Maladies Respiratoires, Lille, France.

CORRESPONDENCE

$\mathrm{C}-\mathrm{H}$. Marquette

Clinique des Maladies Respiratoires Hôpital Albert Calmette

CHRU de Lille

59037 Lille cedex

France

Fax: 33320445611

E-mail: c-marquette@chru-lille.fr

Received:

September 072005

Accepted after revision:

October 312005

SUPPORT STATEMENT

$\mathrm{C}-\mathrm{H}$. Marquette has received

$€ 1,000$ as funds for research during

the previous 5 yrs from William Cook

Europe, Bjaeverskov, Denmark. 
Prospective comparative studies [10-12] showed that a significant amount of the healthcare expenditure related to PSP management could be saved if MA was used as the first step for air evacuation. Simple MA followed by immediate catheter removal proved safe and effective in up to $60 \%$ of the patients [10-12] and allowed half of the study population to be managed in the emergency room only, without further hospitalisation [12]. Nevertheless, with this rational and costsaving approach, nearly half of all patients are hospitalised and result in undergoing a standard anterior or axillary tube thoracostomy [10-12]. An alternative approach that integrated the lack of invasiveness along with the outpatient character and the low cost of MA, while avoiding unnecessary discomfort and costs related to secondary tube thoracostomy for those patients who failed MA, would be a worthwhile development [4].

The use of small-calibre, indwelling catheters attached to a one-way valve or to a water-seal device, instead of the intravenous or thoracocentesis needle catheter, allows air evacuation to continue if the initial MA attempts fail. Such small catheters have proved successful in mixed pneumothorax (PTX) populations, most of them being iatrogenic [1324]. The purpose of the present study was to assess the feasibility, safety and effectiveness of a comprehensive therapeutic algorithm that used small-calibre catheters connected to a one-way valve in first episodes of PSP. The current authors also wanted to see whether the recent BTS and ACCP recommendations for chest tube management $[4,5]$ apply to such small-calibre catheters.

\section{MATERIALS AND METHODS \\ Setting}

The study took place in the Emergency Department and the Department of Respiratory Diseases of the Centre Hospitalier Universitaire (Lille, France), located in an urban area of $\sim 1$ million inhabitants, totalling 72,000 emergency room admissions per year.

\section{Study population and inclusion criteria}

Patients with a first episode of PSP were eligible for inclusion if either of the following applied: 1) complaints of breathlessness or chest pain, regardless of the size of the PTX; or 2) if, on the plain posterio-anterior radiograph, the PTX was considered "large" according to the ACCP definition (distance from apex to cupola $\geqslant 3 \mathrm{~cm}[4]$ ) or to the BTS definition (presence of a visible rim of air $\geqslant 2 \mathrm{~cm}$ between the lung margin and the chest wall [5]). Patients were excluded if they had a history of PTX, whatever the side, or with an underlying lung disease.

Verbal informed consent was requested from patients in order to participate in the study. The protocol was approved by the present authors' institutional Ethical Committee (Comité Consultatif de Protection des Personnes dans la Recherche Biomédicale de Lille, France).

\section{Procedures and protocol}

The catheters were inserted in the second or third anterior intercostal space on the midclavicular line. The whole procedure was performed at the bedside, with the patient in the semi-recumbent position, without fluoroscopic or ultrasound guidance.
A Fuhrman pleural/pneumopericardial drainage set with a Heimlich valve (references C-PPD-850 and C-CASP-A-FORD; COOK ${ }^{\circledR}$ critical care, Bloomington, IN, USA) were used throughout the study. The Fuhrman catheter is an $8.5 \mathrm{~F}$ distally multiperforated polyurethane pigtail catheter, which is commonly marketed for evacuation of air from the pericardial sac or to drain fluid from the pleural space in neonates and infants. Its design enables the insertion of the catheter with the Seldinger technique.

The procedure was carried out aseptically at the bedside following the usual steps of the guidewire tube thoracostomy procedure (Seldinger technique). The Heimlich valve was attached to the catheter with a three-way stopcock inserted between the catheter and the valve. A folded sterile gauze was placed "as a pillow" under the catheter in order to avoid inadvertent kinking (fig. 1) and the insertion point, the gauze and most of the catheter length were sealed with transparent adhesive dressings. Additional adhesive tapes were used to secure the Heimlich valve on the skin. If the patient did not

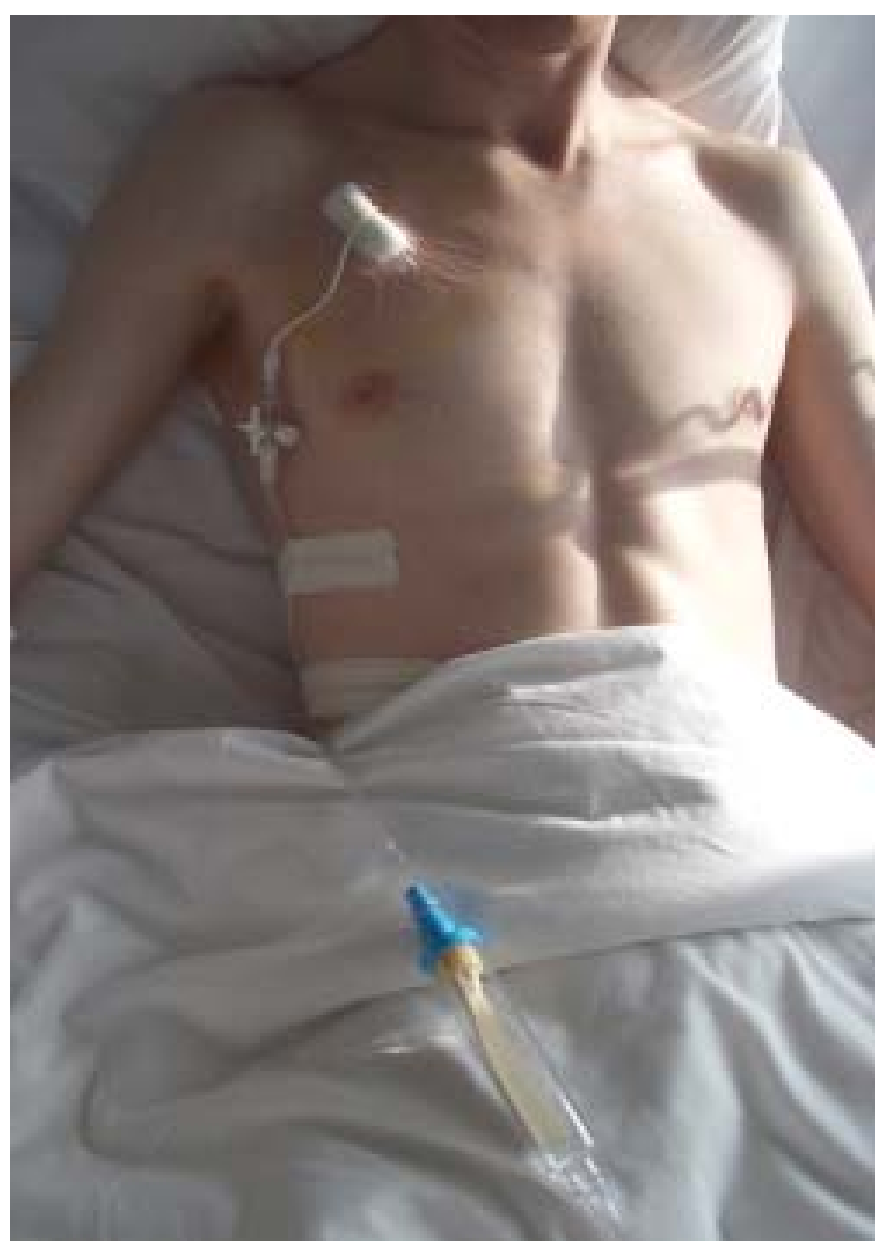

FIGURE 1. A small bore Fuhrman catheter inserted in the third anterior intercostal space on the midclavicular line. A three-way stopcock is inserted between the catheter and the valve. This can be used to flush sterile saline through the catheter if occlusion is suspected. Transparent adhesive plaster bandage seals the insertion point, the gauze and most of the catheter length. Adhesive tape secures the Heimlich valve on the skin. This system enables the patient to be ambulatory and to take a shower. 
tolerate the PTX (dyspnoea), the attending physician could decide to connect the valve to a four-chamber suction system set at -10 to $-20 \mathrm{cmH}_{2} \mathrm{O}$.

A radiograph was taken immediately after the catheter was fitted, to check correct placement of the catheter, and at $6 \mathrm{~h}$ and daily thereafter. No MA was applied to the system at any time. In case of acute dyspnoea, the nursing staff were instructed to connect the valve to a four-chamber suction device. Complete PTX per se (defined as a PTX with air completely surrounding the lung, i.e. without contact between parietal and visceral pleura) was not considered as an indication for immediate suction.

If, at $24 \mathrm{~h}$, lung expansion was complete, or when only a very small rim of apical air was present, the catheter was withdrawn without clamping and the patient was discharged on the same day (fig. 2). If not, pleural drainage via the one-way valve was continued for an additional period of $24 \mathrm{~h}$, followed by the catheter's withdrawal in cases of success.

If, after $48 \mathrm{~h}$ of drainage, the lung failed to re-expand, a water seal device was substituted for the Heimlich valve and $-10--20 \mathrm{cmH}_{2} \mathrm{O}$ suction was applied. When air bubbling had stopped and a chest radiograph had confirmed lung expansion, the catheter was left at water seal for $24 \mathrm{~h}$. After a followup chest radiograph, the catheter was withdrawn and the patient discharged.

If the air leak persisted for $>2$ days, i.e. no lung re-expansion obtained on the fourth day of hospitalisation, a thoracoscopy with talc pleurodesis or video-assisted thoracic surgery (VATS) was considered.
At any time, prescription of plain relief medication was left to the discretion of the attending physician, with special attention being paid to the need for morphine derivatives.

\section{Definitions, end-points and statistics}

Primary end-points were 24-h and 1-week success rates (success was defined as complete or nearly complete, i.e. only a very small rim of apical air, persistent lung expansion, without the need for any additional procedure and without recurrence) and actuarial 1-yr recurrence rate, defined as the time to ipsilateral recurrence. Recurrence-free survival was calculated from the date of the first episode to the date of recurrence or the last day of follow-up. The Kaplan-Meyer method was used.

Secondary end-points were: 6- and 48-h success rates; safety; patient's discomfort, as assessed by the need for pain relief medications; patient's comfort, as assessed by ambulatory status; ability to take a shower; and length of stay. Demographic and descriptive data are given as mean \pm SD.

As this study was primarily intended to evaluate the efficacy and safety of a serial-steps approach with a single system, no control population (parallel or historical) was included.

\section{RESULTS}

A total of 41 consecutive patients entered the study between February 2004 and May 2005. Baseline characteristics are displayed in table 1 . Thirteen PTX (32\%) could be defined as large according to the ACCP definition only, one $(2 \%)$ according to the BTS definition only and 27 (66\%) according to both definitions. The only case where a PTX showed a

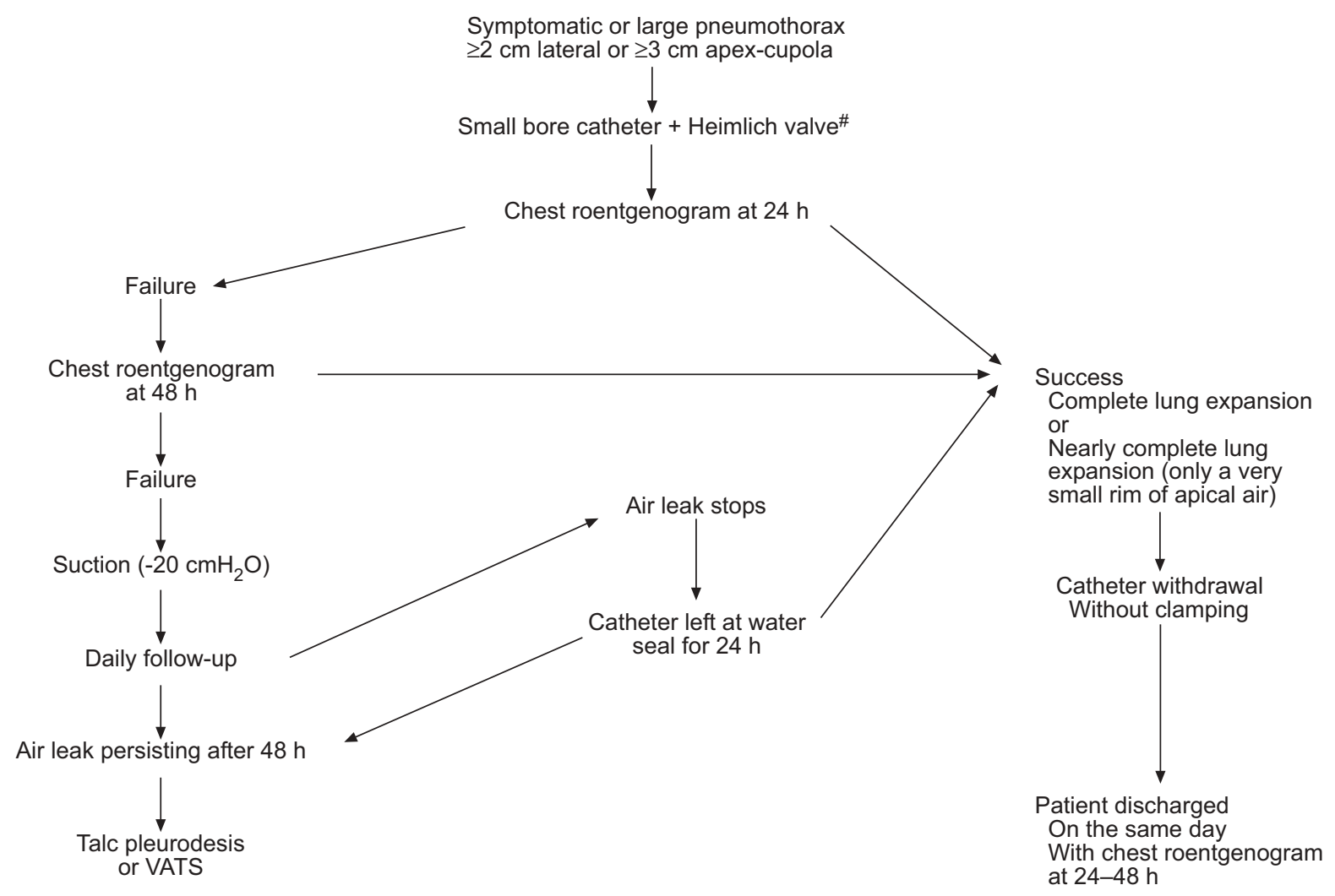

FIGURE 2. Flowchart of therapeutic algorithm. \#: suction could be applied at any time in case of bad tolerance. VATS: video-assisted thoracic surgery. 


\begin{tabular}{|c|c|}
\hline \multicolumn{2}{|l|}{ Characteristic } \\
\hline Sex $M / F$ & $37 / 4$ \\
\hline Age yrs & $26 \pm 6.1(17-40)$ \\
\hline$B M I \mathbf{k g} \cdot \mathbf{m}^{-2}$ & $20.3 \pm 2.8$ \\
\hline Smoking status yes/no & $36 / 5$ \\
\hline Pack-yrs in smokers & $8.1 \pm 7.2$ \\
\hline \multicolumn{2}{|l|}{ "Large" PTX according to } \\
\hline ACCP definition only & $13(32)$ \\
\hline BTS definition only & $1(2)$ \\
\hline ACCP and BTS definition & $27(66)$ \\
\hline Complete PTX \% & $22(54)$ \\
\hline Mediastinal shift \% & $16(39)$ \\
\hline PTX right/left sided & $19 / 22$ \\
\hline First symptoms of PTX occurring at rest/exercise & $33 / 8$ \\
\hline \multicolumn{2}{|l|}{ Pain relief medication during the first $72 \mathrm{~h}$} \\
\hline Acetaminophen & 10 \\
\hline Acetaminophen and NSAID & 4 \\
\hline NSAID & 1 \\
\hline Tramadol & 16 \\
\hline Morphine & 2 \\
\hline
\end{tabular}

Data are presented as $n$, mean $\pm S D$ (range), mean \pm SD or $n(\%)$. M: male; $F$ : female; BMI: body mass index; PTX: pneumothorax; ACCP: American College of Chest Physicians; BTS: British Thoracic Society; NSAID: nonsteroidal antiinflammatory drug.

presence of a visible rim of air $\geqslant 2 \mathrm{~cm}$ between the lung margin and the chest wall without apical PTX had "dependent" PTX with a large rim or air between the lower lobe and the diaphragm. All the catheters were inserted at the bedside by the attending emergency physician without additional fluoroscopic, computed tomography or ultrasound guidance. Insertion was uneventful in all patients, except one who experienced extrathoracically aberrant catheter placement. This patient had a relatively thick chest wall and underwent a second attempt at insertion successfully. Subsequent course in this patient was uneventful.

A total of $22(54 \%)$ patients had a complete PTX, 16 of which showed a mediastinal shift. Tension PTX, defined as a badly tolerated PTX, was observed in two patients; one had total complete PTX with mediastinal shift and the other had only partial PTX without mediastinal shift. As per the protocol, the attending physician applied immediate suction to the catheter (via the valve) in these two cases. Two patients were acutely dyspnoeic on admission (tension PTX). One of them showed complete PTX with mediastinal shift on the chest radiograph. Again, as per the protocol, the attending physician applied immediate suction to the catheter (via the valve) in these two cases.

Protocol violation occurred in three patients. Shortly after catheter insertion, two patients with a complete PTX experienced chest pain, which was interpreted as "bad tolerance" and erroneously led the attending physician to apply immediate suction instead of simple connection of the catheter to the Heimlich valve. One patient with complete lung re-expansion at $48 \mathrm{~h}$ had his catheter clamped for $24 \mathrm{~h}$ before withdrawal. A subsequent course was uneventful.

No accidental catheter dislodgments were observed. A total of $20(48.8 \%)$ patients were able to take a shower within the first $24 \mathrm{~h}$, while having their catheter in place. Eight patients did not require pain relief medications during the first 3 days after catheter placement. In the remaining 33 , only two required morphine, despite a liberal use of this class of drugs in the Clinique des Maladies Respiratoires (Hôpital Albert Calmette, Lille, France; table 1). Of note, the need for morphine in these two patients occurred when wall suction was applied.

Immediate and delayed success rates are displayed in table 2. Analysing the whole group of patients (intention-to-treat analysis) showed that spontaneous lung re-expansion was observed in 15 out of 41 (37\%) patients at $6 \mathrm{~h}$ and in 25 out of $41(61 \%)$ patients at $24 \mathrm{~h}$. Waiting for another $24 \mathrm{~h}$ allowed two additional patients $(66 \%)$ to achieve complete lung expansion. Excluding from analysis the two patients who were inadvertently applied immediate suction did not significantly change the results, as these patients achieved lung re-expansion at 6 and $24 \mathrm{~h}$, respectively. The size of the PTX (complete PTX versus others) did not impact on success rates. Seven out of the 22 patients with complete PTX had their lung re-expanded at $6 \mathrm{~h}$ and 19 at day 7 without the need for any additional procedure and without recurrence.

Failure of PTX to re-expand after $48 \mathrm{~h}$ was observed in 14 patients who then had suction applied to their catheter according to the protocol. This two-steps approach (Heimlich valve first and then suction) was successful in 10 of these patients. Eight of them required 1 day of suction, one required 2 days and one required 4 days of suction. The latter patient was referred for surgery, as well as the four others whose air leaks persisted after 2 days of active suction (fourth day). One underwent a thoracoscopy with talc pleurodesis $(n=1)$, three underwent VATS with bullectomy, subtotal pleurectomy and talc pleurodesis $(n=3)$, and one spontaneously closed the bronchopleural fistula while being on suction, on the fourth day of suction. Hospital stay in the whole group of patients was $3.3 \pm 3.5$ days (range $1-18$ ) and was $2.3 \pm 1.3$ days (range $1-$ 6) when the four patients who underwent surgery were excluded from the calculation.

None of the patients were lost for follow-up. Mean duration of follow-up was $321 \pm 130$ days. Actuarial recurrence rates at 6 months and 1 yr were 22 and $24 \%$, respectively (fig. 3). Two patients had very early ipsilateral PTX recurrence. One who experienced successful re-expansion after $24 \mathrm{~h}$ was readmitted because of PTX recurrence 3 days after discharge. The other

\section{TABLE 2 Study end-points}

$\begin{array}{lc}\text { Success rate } \mathbf{n}=\mathbf{4 1} & \\ 6 \mathrm{~h} & 15(37) \\ 24 \mathrm{~h} & 25(61) \\ 48 \mathrm{~h} & 27(66) \\ 1 \text { week } & 35(85) \\ \text { Actuarial 1-yr recurrence rate \% } & 24\end{array}$

Data are presented as n (\%) unless otherwise stated. 


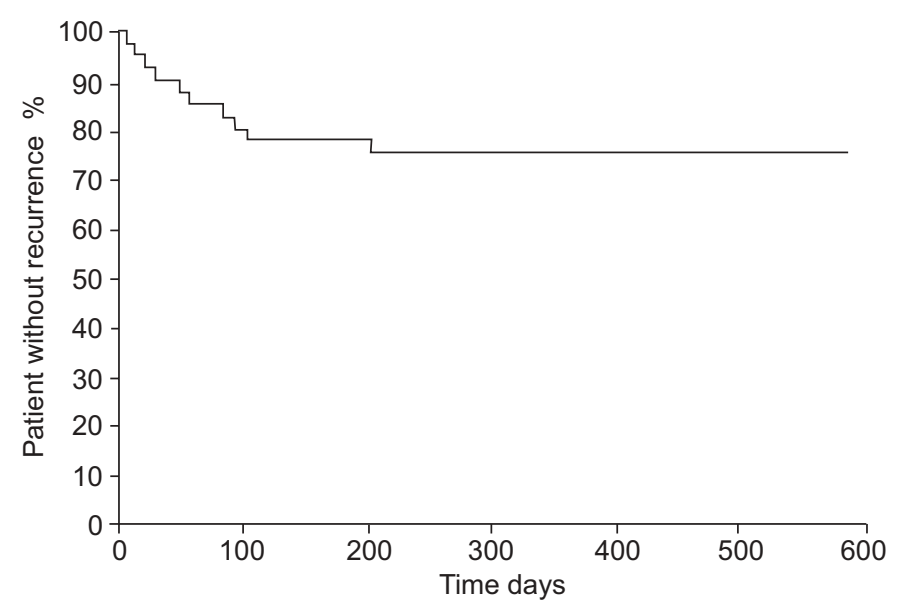

FIGURE 3. Recurrence-free survival

patient, whose lung failed to re-expand with the valve but re-expanded after being placed on suction for 1 day, was readmitted 2 days after discharge. Both patients immediately underwent a thoracoscopy with talc pleurodesis. These two early recurrences and the four cases that required pleurodesis from the very beginning were considered as failures for the calculation of the 1-week success rate.

Eight additional patients experienced later ( $>1$ week) ipsilateral PTX recurrences, at 19 and 20 days, and at 2 (two patients), 3 (three patients) and 6.5 months. One of these eight patients initially underwent thoracoscopy with talc pleurodesis. The mean time to first recurrence was $9 \pm 8.4$ weeks (range 3 days to 6.5 months) and $11 \pm 8.1$ weeks (range 19 days to 6.5 months) if the two very early recurrences were not considered for calculation. Recurrence was minimal in one case (initially treated by thoracoscopy with talc pleurodesis) and was simply observed. One patient refused pleurodesis and was treated conservatively with a simple catheter placed on a valve for 2 days. Out of the six remaining cases, four underwent a thoracoscopy with talc pleurodesis and two underwent VATS.

Two patients experienced contralateral minimal PTX, which did not require hospital admission 5.8 and 7.7 months, respectively, after discharge. These patients were not considered as recurrences for the calculation of the 1-yr success rate.

\section{DISCUSSION}

The present study, conducted in a prospective homogeneous series of 41 consecutive patients with a first episode of large PSP, showed that a "one system, two-steps approach" (smallcalibre catheter connected to a Heimlich valve, with suction added after $48 \mathrm{~h}$ if the lung failed to re-expand), was safe and had high short- and long-term success rates. It also preserved patients' comfort and mobility, allowing early discharge.

There is now a general consensus that a "small" PTX should be observed, provided it is well tolerated and the patient is advised to come back in case of worsening breathlessness [3-5, 25]. Large or badly tolerated PTX should have active treatment involving air evacuation. Definition of a "large" PTX is not uniform $[4,5]$. In the ACCP consensus, PTX was defined as

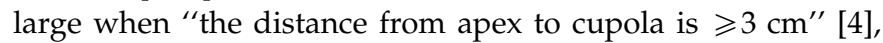
whereas, according to the BTS, a PTX was regarded as large if "a rim of air $\geqslant 2 \mathrm{~cm}$ is visible between the lung margin and the chest wall" [5]. As it remained unclear which of the ACCP and the BTS definitions of a large PTX was the most relevant, the question was deliberately left open to facilitate decision making for the attending physician. Interestingly, the current authors noticed that, in real-life practice, most PTX could be classified as "large" according to the ACCP definition (98\%), whereas only $68 \%$ could be classified as "large" according to the BTS definition. Accordingly, up to $32 \%$ would have been simply observed if the current authors' decision to evacuate air had been based on the BTS definition of large PTX. Since the last ACCP and BTS guidelines were published, this study is the first one to have assessed the size of PTX according to both definitions. From the study's data, it can be inferred that these definitions produce a decision-making conflict in terms of the need for air evacuation, one leading to "overtreatment" and the other to "undertreatment".

Current data suggests that there is little place for recurrence prevention in first PSP episodes and, thus, at least for a first episode, the technique to evacuate the air from the thorax has to be chosen independently of any recurrence prevention technique. Regarding air evacuation, there is now compelling evidence that simple MA should be considered as the primary treatment in uncomplicated PSP [10-12]. This has been taken into account in the recently published guidelines of the BTS [5]. Nonetheless, hospital admission, chest tubes and suction remain the standard approach in many institutions and represent the first step in large-size PSP according to the ACCP guidelines [4].

Conservatism of physicians is certainly not the only factor accounting for the reluctance of physicians to start with MA when first episodes of PSP present themselves. Whereas, in the prospective series, the immediate success of MA averages $72 \%$, real-life clinical practice is less optimistic, with an immediate success closer to $50 \%$. Nevertheless, this allows half the patients to be discharged without an additional overnight stay. However, this does mean that almost half of the patients culminate with a chest tube, after having tried to pump out $\geqslant 2 \mathrm{~L}$ of air from the pleural cavity with a syringe connected to a catheter, which kinks and moves back and forth. Unfortunately, it is difficult to predict failure of MA, except when no resistance is felt after having pumped out $\geqslant 2.5 \mathrm{~L}$ of air [26]. Thus, except when immediate failure is clinically or radiologically noticed, additional observation (3-6 h) is recommended for safety reasons until full re-expansion of the lung is confirmed [4]. In order to avoid prolonged observation after MA, followed by the unnecessary discomfort of secondary tube thoracostomy, many physicians still rely on conventional chest tubes for primary treatment in uncomplicated PSP.

The use of small-calibre catheters was initially reported by radiologists for management of iatrogenic PTX after transthoracic needle biopsies [13-15]. Subsequent studies included first episodes and recurrences of PSP, as well as secondary, traumatic and iatrogenic PTX [16-21]. Except for one study [16], the catheters were catheter-over-needle systems, with no shielding of the needle tip, and were placed either with [13-15, 23 ] or without [16-22, 24] radiological or ultrasound guidance. Not surprisingly, suction applied to these small catheters functioned as well as simple MA. When suction failed to give 
immediate re-expansion, connection to a one-way valve was advised, eventually followed by wall suction if the air leak continued. The failure rate of catheter wall suction when MA had failed ranged from $8-31 \%$ [16, 18-21]. Most of these studies were retrospective and therefore did not follow clear recommendations regarding the stepwise management of underlying air leaks. In contrast, VALLEE et al. [16] followed a standardised approach with a small-calibre catheter in a prospective series of 37 PTX in 35 patients. Half of the cases were spontaneous PTX, but no distinction was made between primary and secondary PTX. A multiperforated polyurethane pigtail catheter, inserted at the bedside with the Seldinger technique, was used. If two MA attempts failed, a Heimlich valve was connected to the catheter and the patient was observed for $1 \mathrm{~h}$. If the PTX persisted, the Heimlich valve was placed at $-20 \mathrm{cmH}_{2} \mathrm{O}$ suction for $1 \mathrm{~h}$. Persistent PTX was then eventually treated by chest tube thoracostomy. Twenty-two cases $(59 \%)$ responded to MA. In the remaining 15 cases, seven (47\%) responded to Heimlich valve attachment, three $(20 \%)$ responded to catheter suction and five (33\%) required a chest tube thoracostomy. A similar approach was followed, using a serial-steps approach with a single system, in a homogeneous population of patients with a first episode of PSP. The time frames recommended in the recent BTS guidelines were also included in the therapeutic algorithm for deciding when to proceed to the next step, i.e. proceed from water-seal to active suction and from suction to thoracoscopy. Lastly, in order to simplify the procedure, and as it was known from the literature that the catheter connected to a Heimlich valve could be sufficient to obtain lung re-expansion [20], the catheter's MA step was deliberately skipped.

Demographic characteristics of the current study's population agree with common findings in PSP, confirming that this disease usually occurs at rest in tall, thin, young, smoking males. It is noteworthy that all the catheters were put in place at the bedside, without any guidance, by $>20$ different practitioners, all of whom were familiar with the Seldinger technique. The majority of the patients were able to walk around within the first $24 \mathrm{~h}$, with half of them being able to take a shower with their catheter. Only two patients, who required wall suction of the catheter, needed morphine for chest pain relief. The catheters could be withdrawn after $24 \mathrm{~h}$ in $61 \%$ of the patients and in two-thirds of the patients after $48 \mathrm{~h}$. At that time (on the second day), if the lung failed to reexpand, the catheter could be used as a conventional tube and active suction applied for another $48 \mathrm{~h}$. In those patients where bubbling was witnessed after $48 \mathrm{~h}$ of active suction (i.e. air leak persisting on the fourth day), surgical referral was considered.

To apply underwater seal drainage, the current authors chose to use a mini Heimlich valve, which, when compared with an underwater seal bottle system, is cheaper, less bulky, does not need to be kept upright, and enables the patient to be more mobile. However, bubbling cannot be visualised with this simple system and, hence, a minimal silent air leak can be missed and the catheter can be removed too early. This is certainly what occurred in the current study's two cases that experienced successful re-expansion followed by early (within 7 days) recurrence. These two cases were thus considered as failures for the calculation of the 1-week success rate and as recurrences for the calculation of the recurrence-free survival.
Despite this, when 24-h and 1-week success rates are taken as end-points, this method, based on a single procedure with a stepwise approach, does as well as simple MA or conventional chest tube thoracotomy [10-12]. In addition, the length of stay compared very well with those reported in recent studies comparing MA and conventional chest tube [10-12].

The current authors are not aware of study recurrence rates using actuarial survival analysis (i.e. recurrence-free survival). Most of the studies provide the crude percentage of recurrence after a variable period of time [12, 21, 27, 28]. Despite this, the recurrence rates observed here agree with current data, showing that recurrence rates after a first episode of PSP treated with an air evacuation technique vary from $16-52 \%$, averaging $30 \%$, with the majority occurring in the first year [29]. Thus, when recurrence of PTX at 12 months is taken as an end-point, the method described here is as effective as MA or conventional chest tube thoracotomy.

The British Thoracic Society recommends the use of smallcalibre catheters as an alternative to manual needle aspiration to reduce the need for repeat aspiration, leaving the catheter in place until full re-expansion of the lung is confirmed [5]. The absence of published data regarding the use of such small catheters in the very context of primary spontaneous pneumothorax may explain why they have not gained wider acceptance. This is the first prospective study integrating recent guidelines showing that a stepwise approach with a small-calibre catheter attached to a Heimlich valve kit could be safely proposed as the first and single treatment in primary spontaneous pneumothorax. This approach, which avoids the unnecessary discomfort related to secondary tube thoracostomy in patients who fail simple manual needle aspiration, allowed most of the patients (37 out of 41 ) to be mobile in hospital during the first $48 \mathrm{~h}$ following the procedure. The current authors' believe that further studies are warranted to assess this "one system, serial-steps approach" in an outpatient management algorithm.

\section{ACKNOWLEDGEMENTS}

The following members of the Pneumothorax Study Group participated in this study: J-F. Poirette, M. Moby, G. Smith (Service d'Accueil et de Traitement des Urgences, Hôpital Roger Salengro, CHRU Lille) and A. Brichet, I. Tillie-Leblond, A. Scherpereel, J-J. Lafitte, A.B. Tonnel (Clinique des Maladies Respiratoires, Hôpital Albert Calmette, CHRU Lille).

\section{REFERENCES}

1 Melton LJ 3rd, Hepper NGG, Offord KP. Incidence of spontaneous pneumothorax in Olmsted County, Minnesota: 1950 to 1974. Am Rev Respir Dis 1979; 120: 1379-1382.

2 Bense L, Eklund G, Wiman LG. Smoking and the increased risk of contracting spontaneous pneumothorax. Chest 1987; 92: 1009-1012.

3 Miller AC, Harvey JE. Guidelines for the management of spontaneous pneumothorax. BMJ 1993; 307: 114-116.

4 Baumann MH, Strange C, Heffner JE, et al. Management of spontaneous pneumothorax. An American College of Chest Physicians Delphi Consensus Statement. Chest 2001; 119: 590-602. 
5 Henry M, Arnold T, Harvey J. BTS guidelines for the management of spontaneous pneumothorax. Thorax 2003; 58: Suppl. 2, 39-52.

6 Baumann $\mathrm{MH}$, Strange C. The clinicians perspective on pneumothorax management. Chest 1997; 112: 822-828.

7 Yeoh JH, Ansari S, Campbell IA. Management of spontaneous pneumothorax - a Welsh survey. Postgrad Med J 2000; 76: 496-500.

8 Baumann MH, Strange C. Treatment of spontaneous pneumothorax: a more aggressive approach? Chest 1997; 112: 789-804.

9 Ponn RB, Siverman HJ, Federico JA. Outpatient chest tube management. Ann Thorac Surg 1997; 64: 1437-1440.

10 British Thoracic Society Research Committee. Comparison of simple aspiration with intercostal drainage in the management of spontaneous pneumothorax. Thorax 1993; 48: 430-431.

11 Andrivert P, Djedaim K, Teboul J-L, et al. Spontaneous pneumothorax: comparison of thoracic drainage vs immediate or delayed needle aspiration. Chest 1995; 108: 335-340.

12 Noppen M, Alexander P, Driesen P, et al. Manual aspiration versus chest tube drainage in first episodes of primary spontaneous pneumothorax. Am J Respir Crit Care Med 2002; 165: 1240-1244.

13 Sargent EN, Turner AF. Emergency treatment of pneumothorax. A simple catheter technique for use in the radiology department. Am J Roentgenol 1970; 109: 531-535.

14 Conces Jr DJ, Tarrer RD, Cory Gray W, et al. Treatment of pneumothoraces utilising small calibre chest tubes. Chest 1988; 94: 55-57.

15 Casola G, vanSonnenberg E, Keightley A, Ho M, Withers C, Lee AS. Pneumothorax: radiologic treatment with small catheters. Radiology 1988; 166: 89-91.

16 Vallee $P$, Sullivan M, Richardson H, Bivins B, Tomlanovich M. Sequential treatment of a simple pneumothorax. Ann Emerg Med 1988; 17: 936-942.

17 Delius RE, Obeid FN, Horst HM, Sorensen VJ, Fath JJ, Bivins BA. Catheter aspiration for simple pneumothorax. Experience with 114 patients. Arch Surg 1989; 124: 833-836.
18 Laub M, Milman N, Müller D. Role of small calibre chest tube drainage for iatrogenic pneumothorax. Thorax 1990; 45: 748-749.

19 Samelson S, Goldberg E, Ferguson M. The thoracic vent: clinical experience with a new device for treating simple pneumothorax. Chest 1991; 100: 880-882.

20 Minami H, Saka H, Senda K, et al. Small caliber catheter drainage for spontaneous pneumothorax. Am J Med Sci 1992; 304: 345-347.

21 Martin T, Fontana G, Olak J, Ferguson M. Use of pleural catheter for the management of simple pneumothorax. Chest 1996; 110: 1169-1172.

22 Roggla M, Wagner A, Brunner C, Roggla G. The management of pneumothorax with the thoracic vent versus conventional intercostal tube drainage. Wien Klin Wochenschr 1996; 108: 330-333.

23 Liu CM, Hang LW, Chen WK. Pigtail tube drainage in the treatment of spontaneous pneumothorax. Am J Emerg Med 2003; 21: 241-244.

24 Vedam H, Barnes DJ. Comparison of large- and small-bore intercostal catheters in the management of spontaneous pneumothorax. Intern Med J 2003; 33: 495-499.

25 Sahn SA, Heffner JE. Spontaneous pneumothorax. N Engl J Med 2000; 342: 868-874.

26 Harvey J, Prescott RJ. Simple aspiration versus intercostal tube drainage for spontaneous pneumothorax in patients with normal lungs. BMJ 1994; 309: 1338-1339.

27 Abdala OA, Levy RR, Bibiloni RH, Viso HD, De Souza M, Satler VH. Advantages of video assisted thoracic surgery in the treatment of spontaneous pneumothorax. Medicina (B Aires) 2001; 61: 157-160.

28 Tschopp JM, Boutin C, Astoul P, et al. Talcage by medical thoracoscopy for primary spontaneous pneumothorax is more cost-effective than drainage: a randomised study. Eur Respir J 2002; 20: 1003-1009.

29 Schramel FM, Postmus PE, Vanderschueren RG. Current aspects of spontaneous pneumothorax. Eur Respir J 1997; 10: $1372-1379$. 